\title{
Shape manipulation using physically based wire deformations
}

\begin{abstract}
This paper develops an efficient, physically based shape manipulation technique. It defines a 3D model with profile curves, and uses spine curves generated from the profile curves to control the motion and global shape of 3D models. Profile and spine curves are changed into profile and spine wires by specifying proper material and geometric properties together with external forces. The underlying physics is introduced to deform profile and spine wires through the closed form solution to ordinary differential equations for axial and bending deformations. With the proposed approach, global shape changes are achieved through manipulating spine wires, and local surface details are created by deforming profile wires. A number of examples are presented to demonstrate the applications of our proposed approach in shape manipulation.
\end{abstract}

Keywords: shape manipulation, surface models, physically based wire deformations, ordinary differential equations, closed form solution.

\section{Introduction}

Shape manipulation is very important in many applications including computer-assisted industrial and artistic design. The traditional shape manipulation is achieved by directly tweaking surface or control points which is effective in local shape manipulation but less efficient in global shape control. The current active shape manipulation methods are space and surface deformation techniques.

Space deformation techniques [1-3] deform 3D models by warping the surrounding space. They are suitable for global shape manipulation. Popular space deformation techniques include free-form deformations initially introduced by Sederberg and Parry [4] and cage-driven deformations. Free-form deformations embed a geometric model in a 3D lattice with a small number of control points. By deforming the lattice, the embedded model deforms giving controllable global deformations. Cage-driven deformations were investigated in some literature such as [5-9]. They enclose a 3D model in a cage with the similar topology and geometry to the model but few polygons. The model is deformed by manipulating the cage. Cage-driven techniques are simple and efficient.

Surface deformation techniques [10-14] treat surface deformations as a global variational optimization problem of the differential properties of the edited surface. According to the survey given in [11], surface deformation techniques can be classified into multiresolution editing and deformation based on differential surface representations. Multiresolution editing techniques [15-17] are based on simplifications and linearizations of the nonlinear thin-shell deformation model. Considering the local differential properties, deformation techniques based on differential surface representations [18$20]$ can create globally smooth and locally detailed high-resolution deformations with the cost in solving a sparse linear system of equations.

The above space and surface deformation techniques did not consider the underlying physics of object deformations although multiresolution editing is based on a thin-shell deformation model. In order to improve the realism, physically-based surface deformations were introduced by Terzopoulos et al. [21] and developed by many researchers [22-25]. Physically-based surface deformations involve heavier numerical calculations and slower responding time compared with geometric deformation techniques.

Shape manipulation techniques based on curve deformations use wires to control surface shapes. Singh and Fiume's wire-based deformation model is influential in the field of wire-based modelling 
[26]. In the work, wires are used to describe the deformations of an object. By specifying an influence domain to a wire, the object is reshaped by deforming the wires. Through representing a surface with a set of curves, significant computational efficiency is gained due to the decoupling of the two surface parameters $u$ and $v$. Although curve based surface deformations become more active in recent years [27-30], how to introduce the underlying physics into curve based surface manipulation for more realistic deformations remains an open problem.

The work presented in this paper aims to tackle this issue. It combines the strengths of curve based surface deformations with the realism of physically-based modelling.

Unlike the existing physically based shape manipulation techniques which operate on surfaces or solids directly with expensive numerical algorithms, we manipulate 3D models through efficient deformations of spine and profile wires. When creating a same deformation, our proposed approach is much simpler and faster than shape manipulation using physics-based surface deformations because our proposed approach is based on analytical calculations of a physically based model.

Different from the wires used for surface manipulation in the existing work which are purely geometric, our proposed wires have material and geometric properties and deform under external forces. The related surface manipulation technique can create more realistic deformations and predict the deformed shape of the objects met in many industrial applications where the material and geometric properties as well as external forces and boundary constraints are known. In contrast, curves based surface manipulation and space and surface deformation techniques are incapable in dealing with these issues.

We will discuss the generation of profile and spine curves in Section 2, the physically based wire deformation model and closed form solution in Section 3, transformation of profile and spine curves into profile and spine wires in Section 4, transfer of wire deformations to 3D models in Section 5 and applications of our proposed approach in Section 6.

\section{Generation of profile and spine curves}

In order to carry out shape manipulation by deforming profile and spine wires, we first represent a 3D surface model with profile curves.
Then spine curves are determined from the profile curves.

\subsection{Profile curves}

Profile curves are a set of curves on the surface of a 3D model which are the most suitable for description of the shape of the surface.

We propose an automatic operation to define profile curves. First, a very rough central curve is drawn within each part of a 3D model. According to the number of profile curves which we want to generate, the curve is divided into the corresponding segments. At the interface point between two adjacent segments, the rays whose number is equal to the points used to define a profile curve are generated. Then we calculate the intersecting points between the rays and the corresponding part of the 3D model and use them to generate the profile curve.

For a goat model shown in Figure 1a, we used 103 profile curves with 30 curves for the head, neck and torso. With the above method, the generated blue profile curves were depicted in Figure $1 b$.

We timed the generation process of all 103 profile curves with different intersecting points on a $1 \mathrm{GHz}$ PC and listed it in Table 1 where NIP stands for the number of intersecting points on a profile curve and $\mathrm{T}$ for the time generating all the profile curves. It can be seen from the table that our proposed automatic operation is very efficient.

Table 1. Time generating 103 profile curves of a goat model

\begin{tabular}{|l|l|l|l|}
\hline NIP & 20 & 50 & 100 \\
\hline $\mathrm{T}$ (seconds) & $2.44 \times 10^{-7}$ & $5.59 \times 10^{-7}$ & $1.12 \times 10^{-6}$ \\
\hline
\end{tabular}

(a)

(b)

Figure 1: Definition of profile curves.

\subsection{Spine curves}

The most important characteristics of shape changes can be represented with spines. We therefore introduce spine curves as one of the most important shape manipulation tools.

To determine a spine curve, we calculate the geometric centre of profile curves. The spine curve is generated by interpolating these geometric centres. Figure $2 \mathrm{a}$ and Figure $1 \mathrm{~b}$ show closed profile curves in blue and the corresponding spine curves in pink. Figure $2 b$ indicates a set of open profile curves in blue and their spine curve in red. 


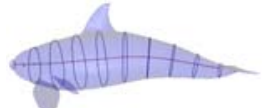

(a)

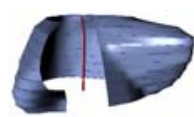

(b)
Figure 2: Determination of the spine curve.

\section{Physically-based model and calculation of wire deformations}

Profile and spine curves can be transformed into the corresponding wires with the methods introduced in Section 4. In this section, we describe the physically-based model and closed form solution of wire deformations.

\subsection{Physically-based model}

Physically based deformations of a wire are tension, compression and bending. Tension or compression is due to axial forces, and bending deformations are caused by bending forces.

Tensile and compressive deformations of a wire are similar to those of an elastic member. Therefore, their governing equation can be derived with the same methodology as that of an elastic member subjected to axial forces, which is a first order ordinary differential equation

$$
E A d \mathbf{X} / d v=\mathbf{P}(v)
$$

where $E$ is Young's modulus, $A$ is the crosssection area of a wire, $\mathbf{X}$ is a vector-valued deformation function, and $\mathbf{P}(v)$ is an axial force.

Young's modulus $E$ is a material property of a wire which indicates the resistance of a material to deformations. The cross-section area $A$ is a geometric property which indicates the resistance of the geometry to axial deformations.

The boundary constraint for tensile or compressive deformations is zero displacement at a specified position $v_{0}$, i. e.,

$$
v=v_{0} \quad \mathbf{X}=0
$$

Bending deformations of a wire are similar to those of an elastic beam. Therefore, their governing equation can be obtained by using the similar treatment to that of an elastic beam under bending forces, which is a fourth order ordinary differential equation depicted below

$$
E I d^{4} \mathbf{X} / d v^{4}=\mathbf{P}(v)
$$

where $E$ is the same as above, i. e., Young's modulus, $\mathbf{P}(v)$ is a bending force, and $I$ is the second moment of area of a wire which indicates the resistance of the geometry to bending deformations.

There are four groups of boundary constraints: simply supported, clamped, and cantilever support with and without rotation at the free end. In order to make physically based wires deformations more flexible, we remove the constraint of zero displacement at supported ends and present the following four groups of modified boundary constraints.

1. Simply supported boundary constraints with position continuity

$$
\begin{array}{lll}
v=0 & \mathbf{X}=\mathbf{X}_{0} & \partial^{2} \mathbf{X} / \partial v^{2}=0 \\
v=1 & \mathbf{X}=\mathbf{X}_{1} & \partial^{2} \mathbf{X} / \partial v^{2}=0
\end{array}
$$

2. Clamped boundary constraints with position and tangent continuities

$$
\begin{array}{lll}
v=0 & \mathbf{X}=\mathbf{X}_{0} & \partial \mathbf{X} / \partial v=0 \\
v=1 & \mathbf{X}=\mathbf{X}_{1} & \partial \mathbf{X} / \partial v=0
\end{array}
$$

3. Cantilever boundary constraints with rotation at the free end

$$
\begin{array}{lll}
v=0 & \mathbf{X}=\mathbf{X}_{0} & \partial \mathbf{X} / \partial v=0 \\
v=1 & \partial^{2} \mathbf{X} / \partial v^{2}=0 & \partial^{3} \mathbf{X} / \partial v^{3}=\mathbf{Q}
\end{array}
$$

where $\mathbf{Q}$ is a shear force acting at the free end. If there is no shear force, $\mathbf{Q}=0$.

4. Cantilever boundary constraints without rotation at the free end

$$
\begin{array}{ccc}
v=0 & \mathbf{X}=\mathbf{X}_{0} & \partial \mathbf{X} / \partial v=0 \\
v=1 & \partial \mathbf{X} / \partial v=0 & \partial^{3} \mathbf{X} / \partial v^{3}=\mathbf{Q}
\end{array}
$$

\subsection{Calculation of axial deformations}

Axial deformations of wires are determined by Eq. (1) subjected to boundary condition (2). Here we discuss how to derive a closed form solution.

Axial forces acting on a wire can be concentrated or line-distributed forces. Linedistributed forces can be changed into concentrated forces by determining the resultant of the linedistributed forces. Due to this reason, we only consider concentrated forces.

Assuming that $J$ concentrated forces $\mathbf{P}_{j}$ act at the position $v_{j}$ of a wire with $v_{j-1}<v_{j}<v_{j+1}$ $(j=1,2,3, \cdots, J)$, the total force is $\sum_{j=1}^{J} \mathbf{P}_{j}$ within $v_{0} \leq v \leq v_{1}, \quad \sum_{j=2}^{J} \mathbf{P}_{j}$ within $v_{1} \leq v \leq v_{2}, \cdots, \sum_{j=J}^{J} \mathbf{P}_{j}$ within $v_{J-1} \leq v \leq v_{J}$ and zero when $v>v_{J}$.

Integrating Eq. (1) within each segment $v_{j} \leq v \leq v_{j+1}$ and summing them up, we obtain a closed form solution of tensile and compressive deformations

$$
\begin{gathered}
\text { For } v_{j} \leq v \leq v_{j+1} \leq v_{J} \quad \mathbf{X}=\left\{\sum_{k=1}^{j}\left[\mathbf{X}\left(v_{k}\right)-\mathbf{X}\left(v_{k-1}\right)\right]\right. \\
\left.\sum_{i=k}^{J} \mathbf{P}_{i}+\left[\mathbf{X}(v)-\mathbf{X}\left(v_{j}\right)\right] \sum_{i=j+1}^{J} \mathbf{P}_{i}\right\} /(E A) \\
\text { For } v \geq v_{J} \quad \mathbf{X}=\left\{\sum_{k=1}^{J}\left[\mathbf{X}\left(v_{k}\right)-\mathbf{X}\left(v_{k-1}\right)\right] \sum_{i=k}^{J} \mathbf{P}_{i}\right\} /(E A)
\end{gathered}
$$


For $v_{0} \leq v \leq v_{1}, j=0$ and the first term in the brace of the right-hand side of the first equation of Eq. (8) is zero. Therefore, the first equation of Eq. (8) becomes

$$
\text { For } v_{0} \leq v \leq v_{1} \quad \mathbf{X}=\left[\mathbf{X}(v)-\mathbf{X}\left(v_{0}\right)\right] \sum_{i=1}^{J} \mathbf{P}_{i} /(E A)
$$

When $v=v_{0}$, Eq. (9) gives $\mathbf{X}=0$. Therefore, the solution given by Eq. (8) satisfies the boundary constraints (2).

With Eq. (8), axial tensile and compressive deformations of wires are obtained analytically.

\subsection{Calculation of bending deformations}

Bending deformations of wires are described by Eq. (3) subject to one of boundary conditions (4-7). In order to achieve high computational efficiency, we develop a closed form solution.

First, we discuss how to represent the bending force $\mathbf{P}(v)$. In general, bending forces can be concentrated or line-distributed lateral forces. With the same treatment as that of line-distributed axial forces, line-distributed lateral forces can be changed into concentrated lateral forces. Therefore, we only consider the treatment of concentrated lateral forces.

If $\mathbf{J}$ concentrated lateral forces $\mathbf{P}_{j}$ act at the position $v_{j} \quad(j=1,2,3, \cdots, J)$, the following mathematical equation is used to represent them

$$
\mathbf{P}(v)=\sum_{n=1}^{\bar{N}} \mathbf{p}_{n} \sin n \pi v
$$

where $\bar{N}$ is total terms of the sine series, and the unknown constant $\mathbf{p}_{n}$ is determined by

$$
\mathbf{p}_{n}=2 \sum_{j=1}^{J} \mathbf{P}_{j} \sin n \pi v_{j}
$$

Substituting Eq. (10) into Eq. (3) and considering boundary conditions (4), the following solution is found

$$
\mathbf{X}(v)=\mathbf{X}_{0}+\left(\mathbf{X}_{1}-\mathbf{X}_{0}\right) v+\sum_{n=1}^{\bar{N}} \mathbf{A}_{n} \sin n \pi v
$$

where

$$
\mathbf{A}_{n}=\mathbf{p}_{n} /\left(n^{4} \pi^{4} E I\right)
$$

Inserting Eq. (10) into Eq. (3) and taking into account boundary conditions (5), we obtain the following solution

$$
\begin{aligned}
\mathbf{X}(v) & =\mathbf{X}_{0}-v \sum_{n=1}^{\bar{N}} \mathbf{B}_{n}-2 v^{2}\left\{1.5\left(X_{0}-X_{1}\right)\right. \\
& \left.-\sum_{n=1}^{\bar{N}}\left[1+0.5(-1)^{n}\right] \mathbf{B}_{n}\right\}+2 v^{3}\left\{\mathbf{X}_{0}-\mathbf{X}_{1}\right. \\
& \left.-0.5 \sum_{n=1}^{\bar{N}}\left[1+(-1)^{n}\right] \mathbf{B}_{n}\right\}+\sum_{n=1}^{\bar{N}} \mathbf{A}_{n} \sin n \pi v
\end{aligned}
$$

where

$$
\mathbf{B}_{n}=n \pi \mathbf{A}_{n}
$$

Introducing Eq. (10) into Eq. (3) and using boundary conditions (6), we achieve

$$
\begin{aligned}
\mathbf{X}(v)= & \mathbf{X}_{0}-v \sum_{n=1}^{\bar{N}} \mathbf{B}_{n}-0.5 v^{2}(1-v / 3)[\mathbf{Q} \\
& \left.+\sum_{n=1}^{\bar{N}}(-1)^{n} n^{2} \pi^{2} \mathbf{B}_{n}\right]+\sum_{n=1}^{\bar{N}} \mathbf{A}_{n} \sin n \pi v
\end{aligned}
$$

Finally, the solution of Eq. (3) after considering the force function (10) and boundary conditions (7) becomes

$$
\begin{aligned}
\mathbf{X}(v) & =\mathbf{X}_{0}-v \sum_{n=1}^{\bar{N}} \mathbf{B}_{n}-0.5 v^{2}\left\{0.5 \mathbf{Q}+\sum_{n=1}^{\bar{N}}\{[-1\right. \\
& \left.\left.\left.+(-1)^{n}\right] n \pi \mathbf{A}_{n}+0.5(-1)^{n} \mathbf{p}_{n} /(n \pi E I)\right\}\right\}+\frac{v^{3}}{6} \\
& {\left[\mathbf{Q}+\sum_{n=1}^{\bar{N}}(-1)^{n} \mathbf{p}_{n} /(n \pi E I)\right]+\sum_{n=1}^{\bar{N}} \mathbf{A}_{n} \sin n \pi v }
\end{aligned}
$$

With the closed form solutions (12), (14), (16) and (17), various bending deformations of wires are determined quickly.

\section{Transformation of profile and spine curves into wires}

Profile and spine curves are purely geometric. They can be changed into profile and spine wires by specifying proper material and geometric properties together with external forces. We address this issue in this section.

\subsection{Profile wires}

Assuming a 3D model and its profile wires are made of the same isotropic materials, the elastic material property of the profile wires is represented by Young's modulus $E$ for axial deformations and the equivalent Young's modulus $E /\left(1-v^{2}\right)$ ( $v$ is Poisson's ratio which is also a material property) for bending deformations as indicated below.

For the axial deformation, the geometric property of wires is the cross-section area $A$. Assuming the thickness of a surface is $h$ and the width of the region covered by a wire is $b$, the geometric property of the wire is determined by $A=b h$.

For the bending deformation, the geometric property is the second moment of area. If a surface has a thickness $h$ and the width of the region covered by a wire is $b$, the bending rigidity is $E b h^{3} /\left[12\left(1-v^{2}\right)\right]$. If we take the equivalent Young's modulus of the wire to be $E /\left(1-v^{2}\right)$, the second moment of area of the profile wire is $b h^{3} / 12$.

The force acting on a profile wire is determined from the force acting on the surface. Then, the force acting on the wire is modified through the 
same deformation as that of the surface at the position of the wire. Assuming the deformation of the surface at the position of a wire is $\mathbf{S}\left(u_{i}, v\right)$ where $u_{i}$ indicates the position of the wire, and the deformation of the wire is $\mathbf{X}(v)$ caused by the force $\mathbf{P}(v)$, we modify $\mathbf{P}(v)$ with a modified coefficient $\xi$ determined by minimising the following error

$$
\bar{E}=\int_{0}^{1}\left\|\mathbf{S}\left(u_{i}, v\right)-\mathbf{X}(v)\right\|^{2} d v
$$

If it is not easy to determine the force acting on a wire from the force acting on the surface, Eq. (18) is used to determine the force $\mathbf{P}(v)$ directly.

We demonstrated the above method by deforming the chest of a chimpanzee model shown in Figure 3a. A deformation region with a complex blue boundary shape was drawn on the chest. Then, the deformation region was parameterized within $0 \leq u \leq 1$ and $0 \leq v \leq 1$. A uniformly distributed lateral force $\mathbf{q}$ is applied in the region. Since the surface deformation is similar to that of an elastic thin plate, the following physically based model was introduced to determine the deformation of the chest surface

$$
E h^{3} /\left[12\left(1-v^{2}\right)\right]\left[\frac{\partial^{2}}{\partial u^{2}}+\frac{\partial^{2}}{\partial v^{2}}\right]^{2} \mathbf{S}(u, v)=\mathbf{q}
$$

where $\mathbf{S}(u, v)$ is a vector-valued function of surface deformation.

Different from the partial differential equations used in $[31,32]$ which have no direct relation with material and geometric properties, Eq. (19) relates bending deformations to material and geometric properties.

In order to maintain smooth transition between the deformed and undeformed regions, the boundary conditions below are applied to Eq. (19).

$$
\begin{array}{lll}
u=0 & \mathbf{X}(u, v)=0 & \frac{\partial \mathbf{X}(u, v)}{\partial u}=0 \\
u=1 & \mathbf{X}(u, v)=0 & \frac{\partial \mathbf{X}(u, v)}{\partial u}=0 \\
v=0 & \mathbf{X}(u, v)=0 & \frac{\partial \mathbf{X}(u, v)}{\partial v}=0 \\
v=1 & \mathbf{X}(u, v)=0 & \frac{\partial \mathbf{X}(u, v)}{\partial v}=0
\end{array}
$$

By solving Eq. (19) subjected to boundary conditions (20), the deformed shape of the chest surface was achieved and depicted in Figure $3 b$.

Our proposed approach can use few profile wires to create the almost same deformed shape as that created with direct physically based surface approach based on Eqs. (19) and (20). For this example, only 7 red profile wires indicated in Figure $3 \mathrm{a}$ were used to create the surface deformation.

From the geometric and material properties of the chest surface, the equivalent Young's modulus and the second moment of area of the profile wires were determined. The force acting on the profile wire $j(j=1,2, \cdots, 5$; and the profile wires $j=0$ and $j=6$ coinciding with the blue boundary curve have no deformation) is $0.5\left(b_{j-1}+b_{j}\right) \mathbf{q}$ where $b_{j-1}$ is the width between the profile wires $j-1$ and $j$, and $b_{j}$ is the width between the profile wires $j$ and $j+1$. Finally, the modified coefficient $\xi$ was determined from Eq. (18) and used to modify the force.

With the developed closed form solution, we obtained the bending deformations of the profile wires which were used to create the chest deformation indicated in Figure 3c. The deformed shapes in Figures $3 \mathrm{~b}$ and $3 \mathrm{c}$ look the same, indicating our proposed approach has the similar computational accuracy to that of physically based surface deformation techniques.

Our proposed surface manipulation technique using physically based wire deformations is very efficient because the obtained closed form solutions can create curve deformations as fast as the other analytical representations of parametric curves such as NURBS. We have timed the process calculating the deformations of different numbers of profile wires on the chest using the same PC as above and the obtained time given in Table 2 where NPS stands for the number of profile curves strongly supports the above argument.

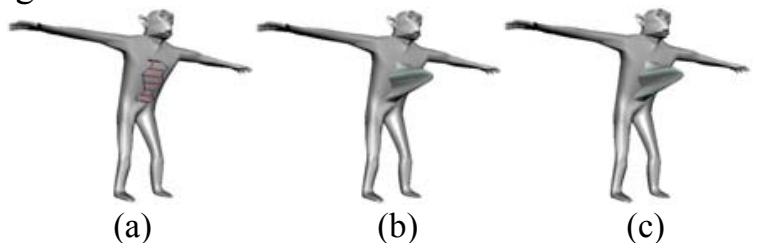

Figure 3. Chest deformation.

Table 2. Time calculating the deformations of profile wires

\begin{tabular}{|l|l|l|l|}
\hline NPS & 100 & 1000 & 10000 \\
\hline $\mathrm{T}$ (seconds) & $1.4 \times 10^{-7}$ & $1.422 \times 10^{-6}$ & $1.429 \times 10^{-5}$ \\
\hline
\end{tabular}

\section{2 spine wires}

The material and geometric properties for rigid and deformable spine wires are different.

The basic motions for rigid spine wires are translation and rotation. More complicated motions are the combinations of these two basic motions. Rigid dynamics is used to describe the motion of rigid bodies.

For translation, the inertia force should be equal to the resultant of all external forces [33]. The inertia force is the product of the mass and acceleration and the mass depends on the density and the volume. Therefore, the material and 
geometric properties for the translation of a spine wire are the density and volume of the corresponding surface, respectively. The resultant force acting on the spine wire is determined by considering the force equilibrium of the surface.

For rotation, the inertia couple should be equal to the resultant torque [33]. The inertia couple is the product of the angular acceleration and mass moment of inertia, and the mass moment of inertia is related to the mass and the radius of rotation. Therefore, the material property of a spine wire is the density of the surface, and the geometric properties are the volume of the surface and the radius of rotation. The resultant torque acting on the spine wire is determined by considering the torque equilibrium.

The deformations for deformable spine wires are axial tension or compression and lateral bending. For material property of deformable spine wires, Young's modulus $E$ is taken to be that of the surface. The geometric properties of a spine curve can be cross-section area or second moment of area which can be determined with the methods of axial deformations of members and bending deformations of beams. Here we take a cylindrical surface as an example and introduce how to determine the geometric properties of its spine wire. Assuming the average radius of the cylindrical surface is $r$ and the thickness of the surface is $h$, the cross-section area of the spine wire for axial deformations is $2 \pi r h$. If a uniformly distributed force $\mathbf{q}$ is applied on the surface thickness in the axial direction, the resultant axial force acting on the spine wire is $2 \pi r h \mathbf{q}$. Using the calculation method of elastic beams, the second moment of area of the above cylindrical surface is $I=\pi r h\left(r^{2}+h^{2} / 2\right)$. If a uniformly distributed lateral force $\mathbf{q}$ acting on the cylindrical surface, equation (10) describing the bending force acting on the spine wire becomes $\mathbf{P}(v)=\sum_{n=1}^{\bar{N}} 2 \mathbf{q}(\sin n \pi v) / n \pi$.

\section{Transfer of wire deformations}

The transfer of wire deformations can be divided into three steps: deformation transfer among spine wires, deformation transfer from spine wires to profile wires, and deformation transfer from profile wires to the 3D model.

Deformation transfer among spine wires is described with the relationship between parent and children. When the parent spine wire deforms, the child spine wires change their positions and orientations accordingly. However, the changes of the child spine wires do not affect the parent spine wire. For example, the deformations of the spine wire of a human torso affect the arms. The shape changes of the upper arm influence the lower arm. The lower arm has an impact on the palm, and the palm has an effect on the fingers.

We realize the deformation transfer from spine wires to profile wires through a binding operation. The profile wires are bounded to their spine wire. When a spine wire is deformed, the profile wires bounded to the spine wire change their positions and orientations.

The deformations of profile wires are transferred to the corresponding 3D model through the following method.

From the undeformed 3D model, we determine the position of surface points at a profile wire or relative to two adjacent profile wires. After profile wires are deformed, we use this position to determine the deformation of surface points as explained below.

If a surface point is on a profile wire, the position of the surface point is determined by simple linear interpolation.

If a surface point $\mathbf{p}_{o}$ is between the $i^{\text {th }}$ profile wire $\mathbf{w}_{i}$ and the $(i+1)^{\text {th }}$ profile wire $\mathbf{w}_{i+1}$ as indicated in Figure 4, we use the following algorithm to relate the surface point $\mathbf{p}_{o}$ to the two profile wires $\mathbf{w}_{i}$ and $\mathbf{w}_{i+1}$.

Assuming that the spine wire determined by a set profile wires including $\mathbf{w}_{i}$ and $\mathbf{w}_{i+1}$ is $\mathbf{w}_{s}$, and the points of the spine wire corresponding to the two profile wires are $\mathbf{p}_{s i}$ and $\mathbf{p}_{s i+1}$, we created a plane from three points $\mathbf{p}_{o}, \mathbf{p}_{s i}$ and $\mathbf{p}_{s i+1}$. Next we calculated intersecting points between the plane and the two profile wires, and obtained two intersecting points $\mathbf{p}_{1}$ and $\mathbf{p}_{2}$ which are the closest to the surface point $\mathbf{p}_{o}$. By making the line $\mathbf{p}_{o} \mathbf{p}_{3}$ perpendicular to the line $\mathbf{p}_{1} \mathbf{p}_{2}$, the position of point $\mathbf{p}_{3}$ was determined

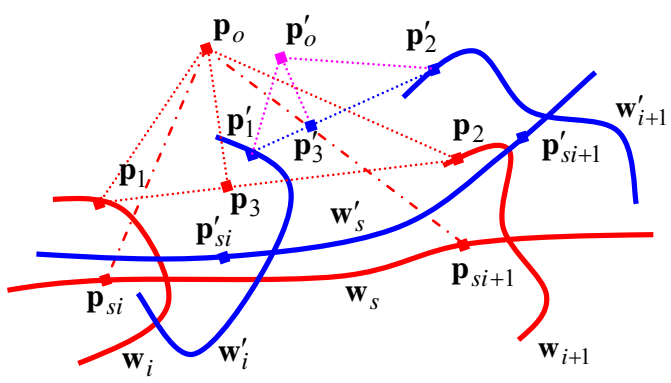

Figure 4: Determination of the new position of surface points between two profile wires.

After deforming the profile wires, spine wire $\mathbf{w}_{s}$ becomes $\mathbf{w}_{s}^{\prime}$, two profile wires $\mathbf{w}_{i}$ and $\mathbf{w}_{i+1}$ are 
changed into $\mathbf{w}_{i}^{\prime}$ and $\mathbf{w}_{i+1}^{\prime}$, and the intersecting points $\mathbf{p}_{1}$ and $\mathbf{p}_{2}$ move to $\mathbf{p}_{1}^{\prime}$ and $\mathbf{p}_{2}^{\prime}$.

In order to determine the new position of surface point $\mathbf{p}_{o}$, we first find the new position $\mathbf{p}_{3}^{\prime}$ of point $\mathbf{p}_{3}$ through $\overline{\mathbf{p}_{1}^{\prime} \mathbf{p}_{3}^{\prime}} / \overline{\mathbf{p}_{1} \mathbf{p}_{3}}=\overline{\mathbf{p}_{1}^{\prime} \mathbf{p}_{2}^{\prime}} / \overline{\mathbf{p}_{1} \mathbf{p}_{2}}$. Then we draw a line through point $\mathbf{p}_{3}^{\prime}$ and perpendicular to the line $\mathbf{p}_{1}^{\prime} \mathbf{p}_{2}^{\prime}$. Finally, we determine the new position $\mathbf{p}_{o}^{\prime}$ of point $\mathbf{p}_{o}$ according to the similarity between triangles $\mathbf{p}_{o} \mathbf{p}_{1} \mathbf{p}_{2}$ and $\mathbf{p}_{o}^{\prime} \mathbf{p}_{1}^{\prime} \mathbf{p}_{2}^{\prime}$.

\section{Shape manipulation applications}

By deforming profile and spine wires using the above physically-based method, one can manipulate complex 3D models efficiently.

\subsection{Shape manipulation through physically based deformations of spine wires}

First, we consider shape manipulation through the axial deformations of spine wires. A candle is used to serve this purpose. The candle was divided into 100 equal segments with 101 nodes leading to $J=100$ in Eq. (8).

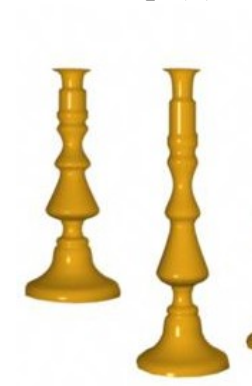

(a) (b)

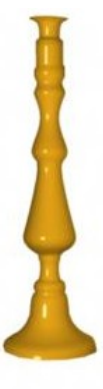

(c)

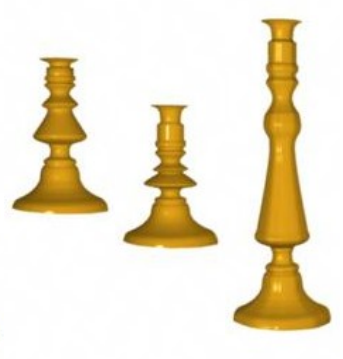

(f)
Figure 5: Tensile and compressive deformations.

First, the upper boundary of the candle was fixed, and an equal downward tensile force is applied at each node. The deformed candle is depicted in Figure $5 \mathrm{~b}$ where Figure $5 \mathrm{a}$ is the original shape. Then we fixed the middle position of the candle and applied an equal upward tensile force at each node of the upper part and a downward tensile force with the same size at each node of the lower part. The produced candle deformation is given in Figure 5c.

Figures $5 \mathrm{~d}$ and $5 \mathrm{e}$ give compressive deformations from one direction and from two opposite directions, respectively. Figure $5 \mathrm{f}$ gives the tensile deformation in a local middle region.

These images indicate that different deformed shapes can be easily achieved by applying different axial forces or choosing different deformation regions.
Bending deformations of a spine wire from simply supported and clamped boundary constraints are shown in Figure 6 where Figure $6 \mathrm{a}$ is the original model, Figure $6 \mathrm{~b}$ is from simply supported boundary constraints with position continuity only, Figure $6 \mathrm{c}$ is from clamped boundary constraints with position and tangent continuities, and Figures 6d, 6e and $6 \mathrm{f}$ are local views which correspond to Figures $6 \mathrm{a}, 6 \mathrm{~b}$ and $6 \mathrm{c}$, respectively.

The bending force used in Figure $6 \mathrm{~b}$ is only half that used in Figure 6c. However, due to free rotation at two boundary nodes, Figure $6 \mathrm{~b}$ has more significant deformations than Figure $6 \mathrm{c}$. The tangential discontinuity can be clearly seen from Figure 6e. In contrast, good tangential continuity is demonstrated by Figure 6f.

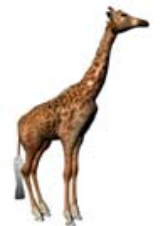

(a)

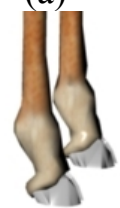

(d)

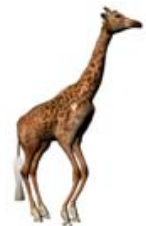

(b)

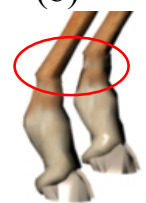

(e)

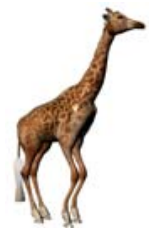

(c)

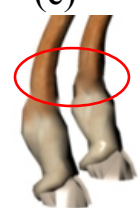

(f)
Figure 6: Bending deformations with simply supported and clamped boundary constraints.

In Figure 7, we demonstrate bending deformations of the spine wire of a trunk model with cantilever boundary constraints where Figure $7 \mathrm{a}$ gives the original model, Figure $7 \mathrm{~b}$ is from cantilever boundary constraints without rotation at the free end and Figure $7 \mathrm{c}$ is from cantilever boundary constraints with rotation at the free end.

The force used in Figure $7 \mathrm{c}$ is also half that used in Figure $7 \mathrm{~b}$. Since the rotation at the upper boundary in Figure $7 \mathrm{c}$ is not subject to any constraints, more noticeable deformation arises.

The above examples demonstrate the effectiveness of spine wires in manipulating global shapes. The images in Figures 6 and 7 indicate that different boundary constraints of spine wires create different bending deformations.

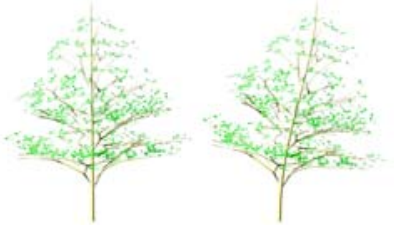

(a)

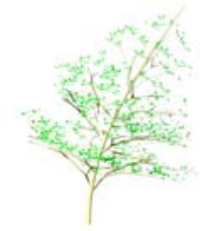

(c)
Figure 7: Bending deformations with cantilever boundary constraints. 


\subsection{Shape manipulation through physically based deformations of profile wires}

Local deformations are achieved by deforming profile wires. Most deformations of profile wires are bending deformations.

As indicated by the lamp post in Figure 8 where Figure $8 \mathrm{a}$ is the original model, all bending deformations of profile wires can be classified into following four groups: (1). deforming the whole length of all profile wires (Figure 8b), (2). deforming a part of all profile wires (Figure 8c), (3). deforming the whole length of some profile wires (Figure 8d), and (4). deforming a part of some profile wires (Figure 8e).

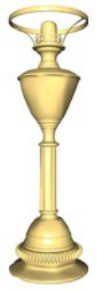

(a)

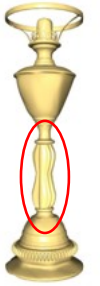

(b)

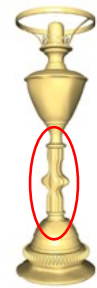

(c)

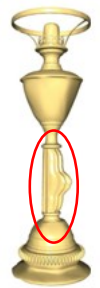

(d)

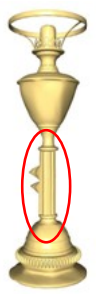

(e)
Figure 8: Four different deformations of a lamp post.

In Figure 9, we demonstrate the deformation created on the upper middle part of a car model. We drew a boundary curve on the model to specify a deformation region. The deformation within the region was described by 7 profile curves. With Eq. (15), we calculated the deformation. Three snapshots taken from the accompanied video were depicted in Figure 9 where the left image indicates the original model and the middle and right images are deformed shapes.

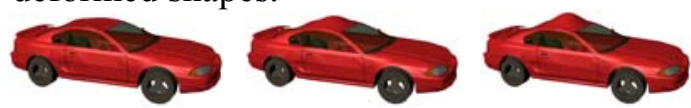

Figure 9: Deformation of the car roof.

\subsection{Combined examples}

By combining physically based deformations of profile and spine wires together, we can create complicated shapes of 3D models.

Our first example is to manipulate a chair model through physically based wire deformations. The original model is shown in Figure 10a, and the deformed chair model is given in Figure 10b.

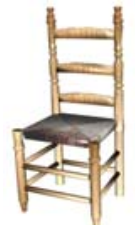

(a)

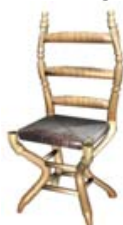

(b)
Figure 10: shape manipulation of a chair model.
Our second example is to manipulate a human model and change it from one gender to another gender and from an elder age to a younger age through physically based deformations of spine and profile wires.

Figure $11 \mathrm{a}$ is an original male model with the profile and spine wires shown in Figure 12a. His pose is altered mainly by the deformations of the spine wires to create the shape in Figure $11 \mathrm{~b}$ with the profile and spine wires indicated in Figure $12 \mathrm{~b}$. A gender change is through physically based deformations of the profile wires to generate the female model in Figure 11c. Then, spine and profile wires are manipulated to convert her pose to that in Figure 11d whose profile and spine wires are depicted in Figure 12c. After deforming her down to the size of the child, further deformations are applied to achieve the child model in Figure 11e. Similar operations are applied to produce the new pose in Figure 11f, and deform the child model in Figure 11f to the female model in Figure $11 \mathrm{~g}$.

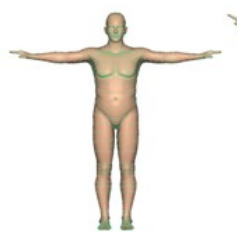

(a)

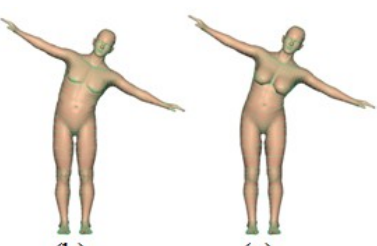

(b) (c)

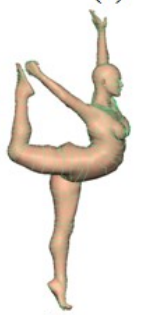

(d)

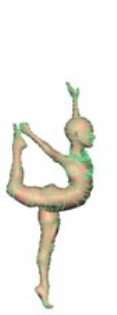

(e)

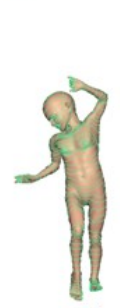

(f)

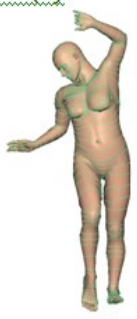

(g)
Figure 11: Shape manipulation of a human model

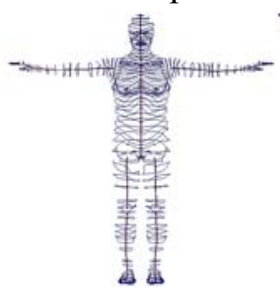

(a)

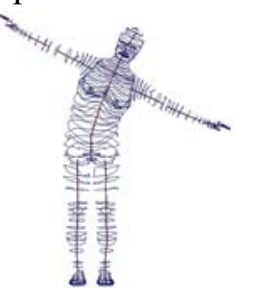

(b)

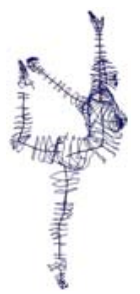

(c)
Figure 12: Profile and spine wires

Our third example is to create the animation of a female model through our proposed shape manipulation method. This example demonstrates how to animate articulated models through our proposed method. The spine wires are rigid and act as skeletons of the female model. Physically based deformations of profile wires are used to achieve shape changes of the skin surface. Some snapshots of the animated female model were depicted in Figure 12. 


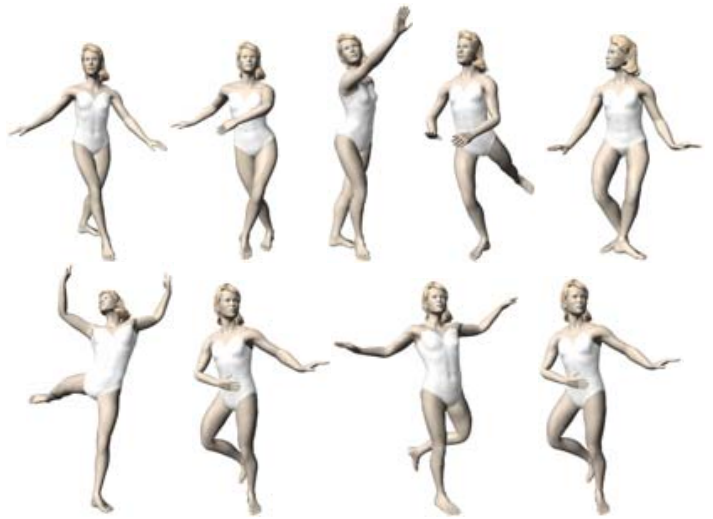

Figure 12: Animation of an articulated model

Our last example is to animate a molluscous model and demonstrate the application of deformable spine wires. With our proposed approach, the shape of an octopus at different poses was obtained. Some deformed shapes taken from the accompanied video were given in Figure 13.

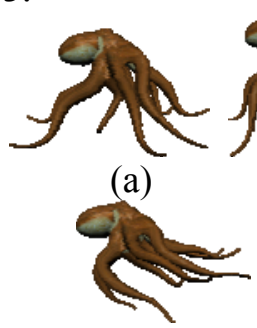

(f) (c)

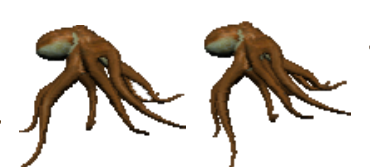

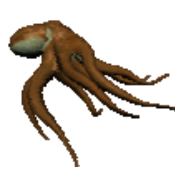

(e) (b)

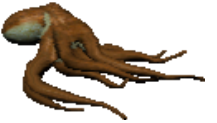

(g)

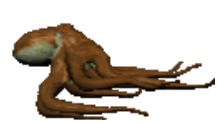

(h)
Figure 13: Animation of a molluscous model

\section{Conclusions and Discussion}

In this paper, an efficient, physically-based shape manipulation technique has been developed. We investigated how to generate profile and spine curves and transform these curves into profile and spine wires. We proposed the physically based model of wire deformations, developed a closed form solution of the model, and examined how to transfer wire deformations to 3D models. Some shape manipulation examples were presented to demonstrate applications of our proposed approach.

Shape manipulation through physically based wire deformations is a novel technique. There are some issues to be investigated in the future.

Our proposed automatic operation of profile curves is very efficient, but does not always generate the most suitable profile curves because it does not take full advantage of the shape changes of 3D models. Due to this reason, the profile curves depicted in Figures $1 \mathrm{~b}$ and 12 can be reduced significantly for the same shape definition and manipulation of $3 \mathrm{D}$ models. It has been demonstrated in Figure 3 that our proposed approach can create complex surface deformations with few profile curves. In our further work, we intend to investigate shape changes of $3 \mathrm{D}$ models and develop a better automatic operation of profile curves.

The second issue is how to maintain the volume of an object. A simple treatment is possible to provide approximate solutions. If the length of the spine wire is constant during shape manipulation, we keep the volume between two adjacent profile wires unchanged before and after their deformation. If the length of the spine wire changes during shape manipulation, we increase or decrease the volume encircled by profile wires to keep the same volume.

The third issue concerning with the deformation of spine wires is surface intersection. If the curvature radius of a spine wire is smaller than the distance between the spine wire and the surface, intersection will arise causing unrealistic shape alteration. To tackle this problem, proper warnings should be given so that the modeler is made aware of the possible anomaly and can take proper actions accordingly.

\section{Acknowledgements}

Some mesh data used in this paper were made available by '500 3D-Objects' (http://www. taschen. com) and free software 'MakeHuman' (http://www. makehuman. org/blog/index.php).

\section{References}

[1] D. Cohen-Or. Space deformations, surface deformations and the opportunities in-between. Journal of Computer Science and Technology, 24(1):2-5, 2009

[2] R. W. Sumner, J. Schmid and M. Pauly. Embedded deformation for shape manipulation. ACM Transactions on Graphics (SIGGRAPH), 26(3): 80(1-7), 2007

[3] M. Botsch, M. Pauly, M. Wicke and M. Gross. Adaptive space deformations based on rigid cells. Computer Graphics Forum (Eurographics), 26(3):339-347, 2007

[4] T. W. Sederberg and S. R. Parry. Free-form deformation of solid geometric models. Computer Graphics (SIGGRAPH), 20(4):151160, 1986

[5] M. S. Floater. Mean value coordinates. Computer Aided Geometric Design, 20(1):1927. 2003

[6] T. Ju, S. Schaefer and J. Warren. Mean value coordinates for closed triangular meshes. ACM 
Transactions on Graphics (SIGGRAPH), 24(3):561-566, 2005

[7] P. Joshi, M. Meyer, T. DeRose, B. Green and T. Sanocki. Harmonic coordinates for character articulation. ACM Transactions on Graphics (SIGGRAPH), 26(3): 71(1-10), 2007.

[8] T. Ju, Q.-Y. Zhou, M. van de Panne, D. CohenOr and U. Neumann. Reusable skinning templates using cage-based deformations. ACM Transactions on Graphics (SIGGRAPH), 27(5):122(1-10), 2008

[9] Y. Lipman, D. Levin and D. Cohen-Or. Green coordinates. ACM Transactions on Graphics (SIGGRAPH), 27(3):78(1-10), 2008

[10] M. Botsch and O. Sorkine. On linear variational surface deformation methods. IEEE Transactions on Visualization and Computer Graphics, 14(1):213-230, 2008

[11] T. Igarashi, T. Moscovich and J. F. Hughes. As-rigid-as-possible shape manipulation. ACM Transactions on Graphics (SIGGRAPH), 24(3):1134-1141, 2005

[12] Y. Lipman, D. Cohen-Or, R. Gal and D. Levin. Volume and shape preservation via moving frame manipulation. ACM Transactions on Graphics, 26(1):5(1-14), 2007

[13] L. Shi, Y. Yu, N. Bell and W.-W. Feng, A fast multigrid algorithm for mesh deformation. ACM Transactions on Graphics (SIGGRAPH), 25(3):1108-1117, 2006

[14] O. K. C. Au, H. Fu, C. L. Tai and D. Cohen-Or. Handle-aware isolines for scalable shape editing. ACM Transactions on Graphics (SIGGRAPH), 26(3):83(1-10), 2007

[15] D. Zorin, P. Schröder and W. Sweldens. Interactive multiresolution mesh editing. Proc. SIGGRAPH, 259-268, 1997

[16] L. Kobbelt, S. Campagna, J. Vorsatz and H.-P. Seidel. Interactive multi-resolution modeling on arbitrary meshes. Proc. SIGGRAPH, 105-114, 1998

[17] M. Marinov and L. Kobbelt. Automatic generation of structure preserving multiresolution models. Computer Graphics Forum (Eurographics), 24(3): 479-486, 2005

[18] Y. Yu, K. Zhou, D. Xu, X. Shi, H. Bao, B. Guo and H.-Y. Shum. Mesh editing with Poisson-based gradient field manipulation. ACM Transactions on Graphics (SIGGRAPH), 23(3):644-651, 2004

[19] Y. Lipman, O. Sorkine, D. Levin and D. Cohen-Or. Linear rotation-Invariant coordinates for meshes. ACM Transactions on Graphics (SIGGRAPH), 24(3):479-487, 2005

[20] A. Nealen, O. Sorkine, M. Alexa and D. Cohen-Or. A sketch-based interface for detailpreserving mesh editing. ACM Transactions on
Graphics (SIGGRAPH), 24(3):1142-1147, 2005

[21] D. Terzopoulos, J. Platt, A. Barr and K. Fleischer. Elastically deformable models. Computer Graphics (SIGGRAP) 21(4):205214, 1987.

[22] A. Nealen, M. Müller, R. Keiser, E. Boxerman and M. Carlson. Physically based deformable models in computer graphics. Computer Graphics Forum, 25(4):809-836, 2006

[23] K. T. McDonnell and H. Qin. A novel framework for physically based sculpting and animation of free-form solids. The Visual Computer, 23(4):285-296, 2007

[24] G. Irving, C. Schroeder and R. Fedkiw. Volume conserving finite element simulations of deformable models. ACM Transactions on Graphics (SIGGRAPH), 26(3):13(1-6), 2007

[25] M. Nesme, P. G. Kry, L. Jeřábková and F. Faure. Preserving topology and elasticity for embedded deformable models. ACM Transactions on Graphics (SIGGRAPH), 28(3):52(1-9), 2009

[26] K, Singh and E. Fiume. Wires: a geometric deformation technique. Proc. SIGGRAPH, 405-414, 1998

[27] H. Pyun, H. J. Shin and S. Y. Shin. On extracting the wire curves from multiple face models for facial animation. Computers \& Graphics, 28(5):757-765, 2004

[28] S.-H. Yoon and M.-S. Kim. Sweep-based freeform deformations. Computer Graphics Forum, 25(3):487-496, 2006

[29] A. Nealen, T. Igarashi, O. Sorkine and M. Alexa, FiberMesh: designing freeform surfaces with 3D curves. ACM Transactions on Graphics (SIGGRAPH), 26(3):41(1-9), 2007

[30] R. Gal, O. Sorkine, N. J. Mitra and D. Cohen-Or. iWIRES: an analyze-and-edit approach to shape manipulation. ACM Transactions on Graphics (SIGGRAPH), 28(3):33(1-10), 2009

[31] L. H. You, J. J. Zhang and P. Comninos. Blending surface generation using a fast and accurate analytical solution of $\mathrm{s}$ fourth-order PDE with three shape control parameters. The Visual Computer, 20:199-214, 2004

[32] J. J. Zhang and L. H. You, Fast surface modelling using $6^{\text {th }}$ order PDE. Computer Graphics Forum (Eurograohics), 23(1):311-320, 2004

[33] J. L. Meriam and L. G. Kraige. Engineering Mechanics: Dynamics, fifth Edition, John Wiley \& Sons, Inc., 2002. 